\title{
Efeitos da invasão por Tradescantia zebrina Heynh. sobre regenerantes de plantas arbóreas em um fragmento de floresta estacional semidecidual secundária em Londrina (PR)
}

\author{
Maurício Cruz Mantoani \\ Jézili Dias \\ Mário Luís Orsi \\ José Marcelo Domingues Torezan * \\ PPG em Ciências Biológicas, Universidade Estadual de Londrina \\ Caixa Postal 6001, CCB/BAV, CEP 96051-990, Londrina - PR, Brasil \\ * Autor correspondência \\ torezan@uel.br
}

Submetido em 31/10/2012

Aceito para publicação em 16/04/2013

\section{Resumo}

Consideradas atualmente como uma das principais causas de perda de biodiversidade no planeta, as espécies exóticas invasoras causam sérios problemas para os ecossistemas naturais, acarretando até mesmo extinções locais. Surpreendentemente, muitas invasões têm origens corriqueiras, como o cultivo de plantas ornamentais em jardins. Este estudo buscou verificar os impactos da invasão biológica pela herbácea exótica Tradescantia zebrina Heynh., sobre a regeneração em um fragmento de floresta secundária. Nesse fragmento, o sub-bosque é em parte dominado por T. zebrina e, para testar a hipótese de que a mesma é responsável pela limitação da regeneração de espécies arbóreas nativas, foram implantadas parcelas em áreas com e sem a presença da espécie invasora. Todos os indivíduos de espécies arbóreas com altura entre $10 \mathrm{~cm}$ e $1 \mathrm{~m}$ foram registrados. A composição de espécies e a estrutura da regeneração foram comparadas entre os tratamentos. Tradescantia zebrina mostrou-se uma forte competidora, afetando a abundância e riqueza de espécies. Embora já tenham sido reportadas na literatura iniciativas de controle com herbicidas não-seletivos, considera-se urgente a realização de mais estudos sobre possíveis formas de controle de T. zebrina, visando o restabelecimento da vegetação nativa.

Palavras chave: Commelinaceae; Comunidade vegetal; Espécies exóticas invasoras; Regeneração natural

\section{Abstract}

Effects of invasion by Tradescantia zebrina Heynh. on tree species regeneration in a secondary Atlantic Forest fragment, in Londrina (PR). Currently considered as one of the major causes of the loss of biodiversity on the planet, invasive alien species cause serious problems to natural ecosystems, leading many native species to become locally extinct. Surprisingly, invasions can come from ordinary sources, such as home gardens. This study aimed to verify the invasive impact of Tradescantia zebrina Heynh., a exotic ornamental herb, on tree regeneration of a secondary Atlantic Forest fragment where the understory is partly dominated by T. zebrina. To test the hypothesis that T. zebrina is limiting the recruitment of native tree species, plots were placed in non-invaded and invaded sites. All tree seedlings between $10 \mathrm{~cm}$ and $1 \mathrm{~m}$ tall were recorded, and the 
composition and structure of regeneration were compared between treatments. Tradescantia zebrina proved to be a strong competitor because it influenced abundance and species richness. Although there are reports in the literature of using non-selective herbicides, it is urgent to carry out further studies on how to control T. zebrina with the goal of restoring native vegetation.

Key words: Commelinaceae; Invasive alien species; Natural regeneration; Vegetal community

\section{Introdução}

Invasões biológicas causam sérios riscos à biodiversidade do planeta, sendo atualmente consideradas como uma das grandes causas de perda de biodiversidade e extinção local de espécies, e ocorrem quando uma espécie exótica invasora coloniza um novo ambiente, alterando características do ecossistema local (MACK et al., 2000). É sabido que grande parte das invasões deriva de distúrbios provocados pela presença do homem, entretanto, mesmo com o baixo registro de invasões em ambientes pouco perturbados (e.g., florestas tropicais) (REJMÁNEK, 1996), plantas exóticas invasoras, principalmente espécies com tolerância ao sombreamento, podem transformar florestas nativas em florestas dominadas por uma única espécie, pela exclusão de regenerantes do sub-bosque, alterações de processos do ecossistema, mudança na composição de árvores do dossel e na estrutura das comunidades invadidas (FINE, 2002).

Muitas invasões têm como fonte atividades corriqueiras, como o cultivo de plantas ornamentais em jardins, onde o material biológico de espécies invasoras pode repousar por anos até que ocorra a contaminação de ambientes naturais. Uma vez que uma população da espécie invasora esteja instalada no habitat natural ou seminatural, pode ser difícil ou mesmo impossível eliminar a população invasora e retornar a vegetação à sua estrutura original (VILÀ; GIMENO, 2007).

A família das Commelinaceae (Monocotiledôneas) abriga grande número de espécies ornamentais largamente cultivadas, que incluem várias reportadas como invasoras que podem escapar de jardins e colonizar ambientes naturais (MARCO et al., 2010). Essas espécies invasoras utilizam rapidamente os recursos disponíveis, aumentando a taxa de crescimento em locais com alta disponibilidade de nutrientes, mesmo sob limitação hídrica (BURNS, 2004; BURNS; WINN, 2006; BURNS et al., 2007).
O gênero Tradescantia contém diversas espécies invasoras espalhadas pelo mundo, como, por exemplo, T. fluminensis Vell. e T. zebrina Heynh. Tradescantia fluminensis causa impactos na regeneração natural em florestas da Nova Zelândia (STANDISH et al., 2001; STANDISH, 2002), alterando a taxa de decomposição de serapilheira e a ciclagem de nutrientes (STANDISH et al., 2004), e também é considerada invasora na Flórida (BURNS; WINN, 2006) e na África do Sul (FOXCROFT et al. 2008).

Já segundo Burns (2004) e os dados do banco de plantas do Serviço de Conservação dos Recursos Naturais dos Estados Unidos (NRCS PLANTS, 2011), T. zebrina estaria causando problemas na Flórida, no Havaí, nas Ilhas Virgens, em Kentucky, na Louisiana e em Porto Rico. No Brasil, há registros de invasão por T. zebrina no Cerrado e na Mata Atlântica (ZENNI; ZILLER, 2011). Carpanezzi (2007) registrou processo de invasão no Parque Estadual de Vila Velha (Ponta Grossa, Paraná) e Rodolfo et al. (2007) registraram a invasão por T. zebrina no Parque Nacional do Iguaçu, relatando que a invasão ocorre a partir das trilhas de visitação. Em Minas Gerais, a espécie estaria prejudicando a regeneração de duas espécies arbóreas nativas (PINTO et al. 2007).

Originária provavelmente do México e países do norte da América Central, de acordo com o banco de dados Tropicos, do Missouri Botanical Garden (http:// www.tropicos.org) e cultivada como planta ornamental em várias partes do mundo, Tradescantia zebrina Heynh. é uma planta herbácea suculenta, conhecida popularmente como trapoeraba-roxa. Suas folhas são verde-arroxeadas, glabras, com duas faixas prateadas brilhantes na face adaxial e roxas na face abaxial. Tem altura entre 15-25 cm, coloniza amplamente áreas com dossel fechado ou meia-sombra e de temperaturas mais quentes. Multiplica-se facilmente por fragmentos de caule, sendo considerada uma "planta daninha" na agricultura (LORENZI; SOUZA, 2008). 
No presente estudo, buscou-se quantificar o efeito da invasão por T. zebrina em um fragmento de floresta secundária, situado dentro do campus da Universidade Estadual de Londrina, testando a hipótese de que a mesma é responsável pela limitação da regeneração de espécies arbóreas nativas.

\section{Material e Métodos}

\section{Área de estudo}

O trabalho foi realizado no campus da Universidade Estadual de Londrina (UEL-2319'19'S e 51¹2’04”W), localizado a sudoeste da cidade de Londrina. Com área total de $2.226 .013 \mathrm{~m}^{2}$, o campus apresenta grande parte de seu território constituído por jardins e gramados. Em todo o campus há presença de árvores nativas e exóticas, e na região sudoeste há dois fragmentos de floresta estacional semidecidual secundária (SHIBATTA et al., 2009).

O presente estudo foi conduzido em um destes fragmentos, com idade aproximada de 25 anos, que apresenta o estrato herbáceo parcialmente dominado por T. zebrina (Figura 1). O estrato superior é composto por uma mistura de espécies nativas e exóticas, incluindo indivíduos remanescentes da floresta original, como

FIGURA 1: Aspecto do interior de floresta estacional semidecidual secundária de 20 anos, em Londrina (PR). Em A, trecho livre de invasão por Tradescantia zebrina Heynh; em B, trecho invadido, com menor densidade de juvenis de espécies lenhosas. As fotografias foram tomadas a partir do mesmo ponto, com $180^{\circ}$ de giro.
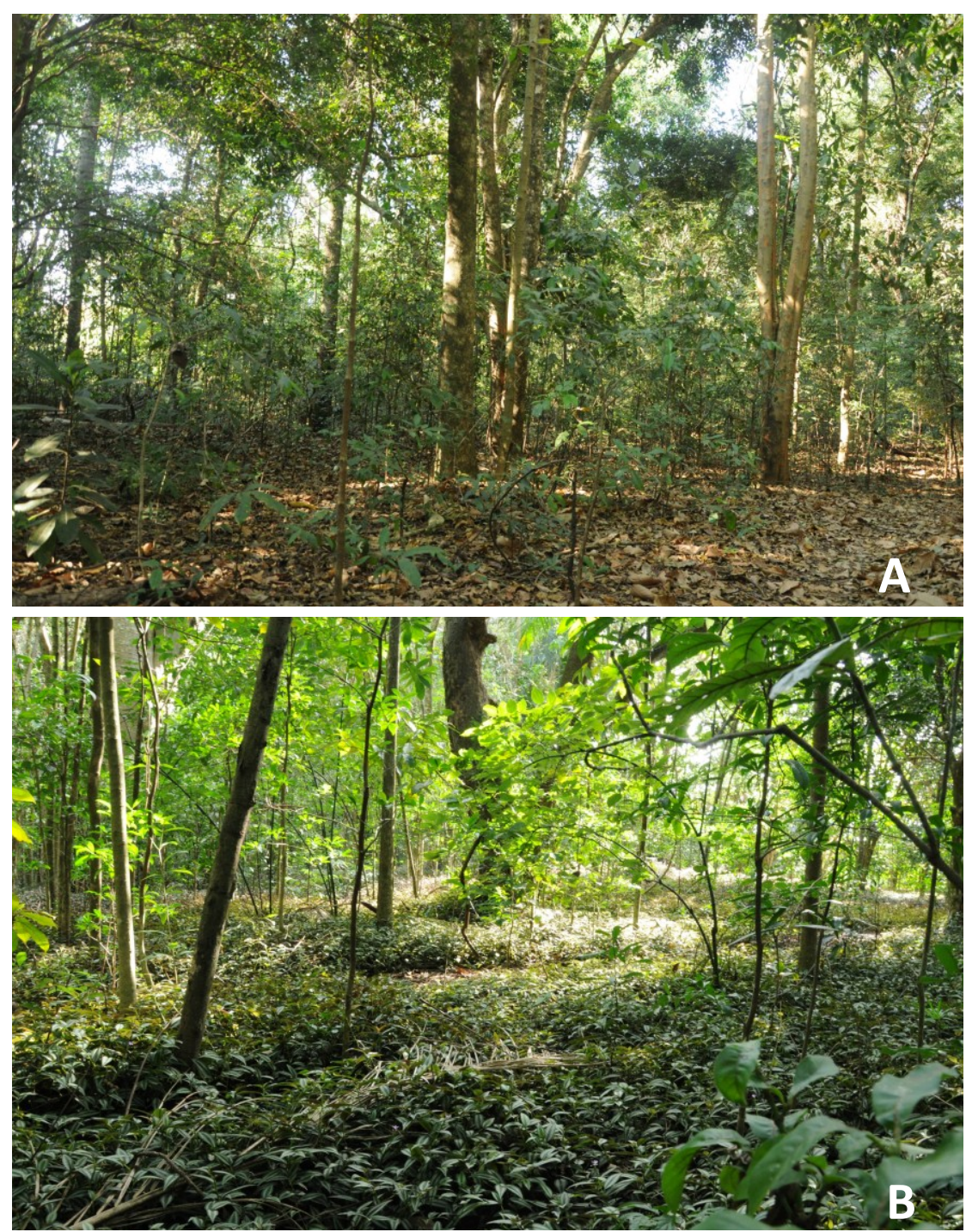
da espécie Aspidosperma polyneuron Müll. Arg., e de espécies exóticas cultivadas nos jardins, que espontaneamente se estabeleceram, como de Magnolia champaca (L.) Baill. ex Pierre. A área não sofre perturbações significativas há 22 anos. Há cerca de dez anos o despejo de entulho de uma reforma junto a uma das bordas trouxe fragmentos de T. zebrina, que passaram a colonizar o local, em direção ao interior do fragmento, a uma taxa aproximada de dois metros por ano (observação pessoal).

\section{Amostragem}

Foram implantadas 60 parcelas de $2 \times 5 \mathrm{~m}$ na área, sendo 30 parcelas na área sem a presença da herbácea exótica (controle) e 30 parcelas na área com T. zebrina (invasão) (Figura 1). Todos os indivíduos de espécies arbóreas com altura maior ou igual a $10 \mathrm{~cm}$ e até 1 $\mathrm{m}$ foram amostrados. A identificação das espécies foi realizada em campo, quando possível, ou por meio de comparações com material do Herbário FUEL. As espécies foram separadas em dois grupos sucessionais (pioneiras/secundárias iniciais e secundárias tardias/ clímax) com base em Cavalheiro et al. (2002) e Zangaro et al. (2003) e também quanto à origem biogeográfica (exóticas e nativas) com base nas informações do banco de dados Tropicos, do Missouri Botanical Garden (http:/www.tropicos.org/NameSearch.aspx), tambérm utilizado como referência para nomenclatura botânica.

A normalidade da distribuição dos dados foi verificada pelo teste de Shapiro-Wilk, e a homocedasticidade pelo teste de Bartlett. Como os dados não atenderam às premissas para o uso de estatística paramétrica, mesmo após transformações, as estimativas de abundância e de riqueza de espécies, para o total da amostragem e para cada grupo foram comparadas entre as parcelas invadidas e não invadidas por meio do teste de Mann-Whitney. Todas as análises foram feitas no software estatístico R v. 2.14.0 (R FOUNDATION FOR STATISTICAL COMPUTING, 2008), adotando-se $\alpha=0,05$.

\section{Resultados e Discussão}

Foram amostrados 2364 indivíduos, distribuídos em 49 espécies (Tabela 1). Desse total, 279 indivíduos (11\% do total) foram classificados em 15 espécies consideradas como exóticas $(30 \%$ das espécies amostradas).

TABELA 1: Lista de espécies arbóreas amostradas em fragmento de floresta estacional semidecidual, no campus da Universidade Estadual de Londrina, em Londrina, PR, com seus respectivos dados de abundância nas áreas com (I) e sem (C) a presença da espécie Tradescantia zebrina. Estágio Sucessionais: P/S - espécies pioneiras/ secundárias iniciais; T/ C - espécies secundárias tardias/ clímax. Origem: E - exótica; N - nativa.

\begin{tabular}{llccccc}
\hline \multirow{2}{*}{ FAMíliA } & \multicolumn{1}{c}{ NOME CIENTÍFICO } & I & C & TOTAL $\begin{array}{c}\text { ESTÁGIO } \\
\text { SUCESSIONAL }\end{array}$ & ORIGEM \\
\hline Anacardiaceae & Mangifera indica L. & 5 & 0 & 5 & $\mathrm{P} / \mathrm{S}$ & $\mathrm{E}$ \\
Annonaceae & Duguetia sp. & 0 & 1 & 1 & $\mathrm{P} / \mathrm{S}$ & $\mathrm{N}$ \\
& Rollinia sylvatica (A. St.-Hil.) Martius & 2 & 7 & 9 & $\mathrm{P} / \mathrm{S}$ & $\mathrm{N}$ \\
Apocynaceae & Aspidosperma polyneuron Müll. Arg. & 46 & 1008 & 1054 & $\mathrm{~T} / \mathrm{C}$ & $\mathrm{N}$ \\
& Tabernaemontana catharinensis (A. DC.) Miers & 11 & 24 & 35 & $\mathrm{P} / \mathrm{S}$ & $\mathrm{N}$ \\
Arecaceae & Euterpe edulis Mart. & 7 & 7 & 14 & $\mathrm{~T} / \mathrm{C}$ & $\mathrm{N}$ \\
& Syagrus romanzoffiana (Cham.) Glassm. & 0 & 4 & 4 & $\mathrm{~T} / \mathrm{C}$ & $\mathrm{N}$ \\
Bignoniaceae & Spathodea campanulata P. Beauv. & 1 & 2 & 3 & $\mathrm{P} / \mathrm{S}$ & $\mathrm{E}$ \\
& Tabebuia roseoalba (Ridl.) Sandwith & 1 & 0 & 1 & $\mathrm{P} / \mathrm{S}$ & $\mathrm{N}$ \\
Clusiaceae & Garcinia cochinchinensis Choisy & 1 & 0 & 1 & $\mathrm{P} / \mathrm{S}$ & $\mathrm{E}$ \\
Euphorbiaceae & Croton floribundus Spreng. & 2 & 14 & 16 & $\mathrm{P} / \mathrm{S}$ & $\mathrm{N}$ \\
Fabaceae & Bauhinia forficata Link & 21 & 3 & 24 & $\mathrm{P} / \mathrm{S}$ & $\mathrm{N}$
\end{tabular}


Bauhinia variegata $\mathrm{L}$.

Caesalpinia peltophoroides Benth.

$\mathrm{P} / \mathrm{S}$

E

Cassia sp.

Delonix regia (Bojer ex Hook.) Raf.

$\mathrm{P} / \mathrm{S}$

$\mathrm{N}$

$\begin{array}{lll}2 & 2 & 4\end{array}$

$\mathrm{P} / \mathrm{S}$

E

Inga edulis Mart.

Inga marginata Willd.

$\mathrm{P} / \mathrm{S}$

E

$\begin{array}{lll}0 & 1 & 1\end{array}$

$\mathrm{P} / \mathrm{S}$

$\mathrm{N}$

Inga striata Benth.

Machaerium stipitatum (DC.) Vogel

$\begin{array}{lll}4 & 0 & 4\end{array}$

$\mathrm{P} / \mathrm{S}$

$\mathrm{N}$

$3 \quad 50 \quad 53$

$\mathrm{P} / \mathrm{S}$

$\begin{array}{lll}1 & 9 & 10\end{array}$

$\mathrm{P} / \mathrm{S}$

$\mathrm{N}$

Parapiptadenia rigida (Benth.) Brenan

$\begin{array}{lll}2 & 37 & 39\end{array}$

$\mathrm{P} / \mathrm{S} \quad \mathrm{N}$

Schizolobium parahyba (Vell.) S.F. Blake

$\begin{array}{lll}0 & 1 & 1\end{array}$

$\mathrm{P} / \mathrm{S}$

$\mathrm{N}$

$\begin{array}{lll}2 & 4 & 6\end{array}$

$\mathrm{P} / \mathrm{S}$

$\mathrm{E}$

Lauraceae Nectandra megapotamica (Spreng.) Mez

$\begin{array}{lll}64 & 355 & 419\end{array}$

$\mathrm{T} / \mathrm{C}$

$\mathrm{N}$

Ocotea puberula (Reich.) Nees.

$\begin{array}{lll}87 & 117 & 204\end{array}$

$\mathrm{T} / \mathrm{C}$

$\mathrm{N}$

Lauraceae Persea americana Mill.

$8 \quad 26 \quad 34$

$\mathrm{P} / \mathrm{S}$

E

Magnoliaceae Michelia champaca L.

Malvaceae Ceiba speciosa (A. St.-Hil.) Ravenna

$0 \begin{array}{lll}0 & 1 & 1\end{array}$

$\mathrm{P} / \mathrm{S}$

$\mathrm{E}$

$\begin{array}{lll}0 & 3 & 3\end{array}$

Meliaceae

Cabralea canjerana (Vell.) Mart.

$3 \quad 21 \quad 24$

$\mathrm{P} / \mathrm{S}$

$\mathrm{N}$

$\mathrm{T} / \mathrm{C} \quad \mathrm{N}$

Guarea guidonia (L.) Sleumer

$\begin{array}{lll}0 & 26 & 26\end{array}$

$\mathrm{T} / \mathrm{C}$

$\mathrm{N}$

Guarea kuntiana A. Juss

Trichilia casaretii C. DC.

$\mathrm{T} / \mathrm{C}$

$\mathrm{N}$

Trichilia claussenii C. DC.

$\begin{array}{lll}1 & 3 & 4\end{array}$

$\mathrm{T} / \mathrm{C}$

$\mathrm{N}$

Trichilia elegans A. Juss.

$\mathrm{T} / \mathrm{C}$

$\mathrm{N}$

Trichilia pallida Sw.

Monimiaceae Mollinedia sp.

$\begin{array}{lll}1 & 2 & 3\end{array}$

$\mathrm{T} / \mathrm{C}$

$\mathrm{N}$

$\begin{array}{lll}0 & 14 & 14\end{array}$

$\mathrm{T} / \mathrm{C}$

$\mathrm{N}$

$\mathrm{P} / \mathrm{S} \quad \mathrm{N}$

Moraceae

Morus nigra $\mathrm{L}$.

$\mathrm{P} / \mathrm{S}$

E

Sorocea bonplandii (Baill.) W.C. Burger, Lanj. \& Boer $\quad 0 \quad 5 \quad 5$

$\mathrm{T} / \mathrm{C}$

$\mathrm{N}$

Myrtaceae

Campomanesia xanthocarpa O. Berg.

$\begin{array}{lll}1 & 7 & 8\end{array}$

$\mathrm{T} / \mathrm{C}$

$\mathrm{N}$

Eugenia pyriformis Cambess.

$\begin{array}{lll}6 & 5 & 11\end{array}$

$\mathrm{T} / \mathrm{C}$

$\mathrm{N}$

Eugenia uniflora L.

$\begin{array}{lll}12 & 7 & 19\end{array}$

$\mathrm{P} / \mathrm{S}$

$\mathrm{N}$

Myrciaria trunciflora O. Berg

$\begin{array}{lll}0 & 3 & 3\end{array}$

$\mathrm{T} / \mathrm{C}$

$\mathrm{N}$

Psidium guajava L.

$\mathrm{P} / \mathrm{S}$

E

Syzygium cumini (L.) Skeels

$\begin{array}{lll}0 & 2 & 2\end{array}$

$\mathrm{P} / \mathrm{S}$

E

Rosaceae Eriobotrya japonica (Thunb.) Lindl.

$\begin{array}{lll}3 & 15 & 18\end{array}$

$\mathrm{P} / \mathrm{S}$

E

Rubiaceae

Coffea arabica $\mathrm{L}$.

$\begin{array}{lll}0 & 7 & 7\end{array}$

Rutaceae

Citrus limon (L.) Burm. F.

$\begin{array}{lll}1 & 128 & 129\end{array}$

$\mathrm{P} / \mathrm{S}$

E

Murraya paniculata (L.) Jack

$\begin{array}{lll}2 & 63 & 65\end{array}$

$\mathrm{P} / \mathrm{S}$

E

Allophylus edulis (St.-Hil., Juss. \& Camb.) Hier. ex

Sapindaceae Niederl.

$\begin{array}{lll}1 & 3 & 4\end{array}$

$\mathrm{P} / \mathrm{S}$

E

Solanaceae Cestrum intermedium Sendtn.

123

$\mathrm{P} / \mathrm{S}$

$\mathrm{N}$

$\begin{array}{lll}0 & 4 & 4\end{array}$

$\mathrm{P} / \mathrm{S}$ 
Tanto a abundância quanto a riqueza de espécies de modo geral foram fortemente prejudicadas pela presença de T. zebrina (Tabela 2), de maneira similar a relatos anteriores para a congênere $T$. fluminensis (KELLY; SKIPWORTH, 1984; STANDISH et al., 2001; PINTO et al., 2007). As parcelas sem T. zebrina (controle) chegam a ter sete vezes mais indivíduos e 11 espécies a mais do que nas amostras com a presença da espécie invasora. Vale ressaltar que esta relação causal é compatível com as observações de campo dos autores, que acompanharam a chegada, o estabelecimento e a expansão de T zebrina no local, ao longo dos dez anos que precederam a amostragem.

Standish et al. (2001) reportaram que a abundância e riqueza de espécies decaíram exponencialmente com o aumento da biomassa de T. fluminensis, resultando em uma redução de 13 vezes na abundância e de oito vezes na riqueza de espécies na área invadida, quando em comparação a áreas sem a herbácea invasora.

Espécies pioneiras senso lato (pioneiras e secundárias iniciais) e tardias (tardias e clímax) responderam de maneira similar, sendo ambos os grupos afetados pela invasão (Tabelas 1 e 2). Da mesma forma, espécies lenhosas exóticas e nativas mostraram similarmente maior abundância e maior riqueza na área sem a presença de T. zebrina, sugerindo que a invasora não favorece a regeneração de outras espécies exóticas (SIMBERLOFF; HOLLE, 1999), mas prejudica a regeneração de espécies lenhosas indistintamente (Tabelas 1 e 2).

As espécies nativas mais abundantes na regeneração, Aspidosperma polyneuron Müll. Arg. (com 1054 indivíduos) e Nectandra megapotamica (Spreng.) Mez (com 419), sofreram forte redução nas parcelas com a presença de T. zebrina. No entanto, algumas espécies mostraram abundância similar nas parcelas com e sem a exótica, a exemplo de Eugenia uniflora L., E. pyriformis Cambess., Euterpe edulis Mart. e Ocotea puberula (Rich.) Ness (Tabela 1). Tal fato pode estar relacionado com a presença de reservas nutritivas, que podem ajudar as plântulas a superar a competição inicial com a herbácea invasora, sugerindo que em áreas com vegetação arbórea estabelecida, mas com domínio de herbáceas invasoras no sub-bosque, a sucessão ecológica poderia favorecer tais espécies em detrimento das que não possuem reservas nutritivas (HOOPER et al., 2002). No entanto, os dados não indicam se a regeneração destas espécies poderia levar a uma redução da população da invasora no futuro ou mesmo evitar o colapso da estrutura florestal. Para tanto, estudos de longo prazo são necessários.

TABELA 2: Abundância e riqueza de regenerantes de espécies arbóreas em um fragmento de floresta estacional semidecidual secundária, no campus da Universidade Estadual de Londrina, em Londrina, PR. Invasão - parcelas invadidas por Tradescantia zebrina Heynh. $(n=30)$. Controle - parcelas sem invasão $(n=30)$. Letras diferentes significam que os valores são significativamente diferentes - teste de Mann Whitney.

\begin{tabular}{lcccc}
\hline & Invasão & Controle & $\mathbf{U}$ & $\boldsymbol{p}$ \\
\hline Abundância Total & $305 \mathrm{a}$ & $2059 \mathrm{~b}$ & 1,5 & $>0,001$ \\
Riqueza total de espécies & $33 \mathrm{a}$ & $44 \mathrm{~b}$ & 76 & $>0,001$ \\
Abundância de pioneiras & $89 \mathrm{a}$ & $479 \mathrm{~b}$ & 91 & $>0,001$ \\
Riqueza de espécies pioneiras & $24 \mathrm{a}$ & $28 \mathrm{~b}$ & 107,5 & $>0,001$ \\
Abundância de tardias & $16 \mathrm{a}$ & $1580 \mathrm{~b}$ & 2,5 & $>0,001$ \\
Riqueza de espécies tardias & $9 \mathrm{a}$ & $16 \mathrm{~b}$ & 132,5 & $>0,001$ \\
Abundância de espécies exóticas & $24 \mathrm{a}$ & $255 \mathrm{~b}$ & 142,5 & $>0,001$ \\
Riqueza de espécies exóticas & $10 \mathrm{a}$ & $12 \mathrm{~b}$ & 175 & $>0,001$ \\
Abundância de espécies nativas & $281 \mathrm{a}$ & $1804 \mathrm{~b}$ & 1,5 & $>0,001$ \\
Riqueza de espécies nativas & $23 \mathrm{a}$ & $32 \mathrm{~b}$ & 97 & $>0,001$ \\
\hline
\end{tabular}


Bauhinia forficata Link apresentou maior abundância nas parcelas invadidas (21 indivíduos contra três nas parcelas sem invasão). No entanto, este resultado provavelmente está relacionado com a distribuição dos adultos restrita a uma das bordas na área de estudo, bem como à dispersão autocórica desta espécie, o que levaria a uma forte pressão de propágulos junto à área invadida. No entanto, não é possível prever a abundância de B. forficata nas mesmas condições de pressão de propágulos e sem a invasora.

Kelly e Skipworth (1984) estudando T. fluminensis em áreas de densa infestação (camadas de $60 \mathrm{~cm}$ de espessura sobre o solo) reportam ter encontrado grande quantidade de regenerantes muito pequenos embaixo da camada da herbácea e poucos juvenis despontando nas áreas invadidas, o que sugere que a alta mortalidade nas menores classes de tamanho (i.e., logo após a germinação) pode ser um dos principais mecanismos pelos quais o efeito negativo da invasora herbácea se manifesta sobre a regeneração de lenhosas. Por outro lado não pode ser descartada uma depreciação do banco de sementes à medida que a invasão se torna permanente e duradoura (VILÀ; GIMENO, 2007), pela interceptação da chuva de sementes, ou ainda outras formas de interferência sobre a regeneração, como competição por recursos naturais (e.g., luz, nutrientes) e possíveis efeitos alelopáticos, ambos não avaliados neste estudo.

A partir dos resultados, foi possível concluir que T. zebrina Heynh. causa forte prejuízo à regeneração de espécies arbóreas, tanto nativas quanto exóticas, no fragmento estudado, e embora a resposta tenha sido desigual entre as espécies, foi similar entre os grupos sucessionais, sugerindo que os prejuízos são independentes de taxas de crescimento, grau de tolerância à sombra ou tamanho da semente, características em que pioneiras e não pioneiras diferem bastante. A magnitude deste efeito sugere que a floresta pode ser levada ao colapso, pela restrição à reposição da mortalidade de árvores adultas, pela alta competição com as plântulas do sub-bosque.

Embora técnicas baseadas no emprego de herbicidas não-seletivos tenham sido sugeridas na literatura para o controle de T. zebrina (DECHOUM; ZILLER, 2013), é necessário investigar formas de controle menos impactantes em ambiente florestal, visando acelerar o restabelecimento da vegetação nativa, evitando danos colaterais às plantas lenhosas (possibilidade associada ao controle químico não seletivo).

\section{Agradecimentos}

Ao CNPq pelo apoio financeiro (processos 503836/2010-9 e 313854/2009-2 - bolsa de produtividade - JMDT). A Odair do Carmo Pavão pelo apoio no trabalho de campo. A Luís Henrique Vidal por suas críticas e sugestões.

\section{Referências}

BURNS, J. H. A comparison of invasive and noninvasive dayflowers (Commelinaceae) across experimental nutrient and water gradients. Diversity and Distributions, Stellenbosch, v. 10, p. 387-397, 2004. BURNS, J. H.; HALPERN, S. L.; WINN, A. A. A test for a cost of opportunism in invasive species in the Commelinaceae. Biological Invasions, Dordrecht, v. 9, p. 213-225, 2007.

BURNS, J. H.; WINN, A. A. A comparison of plastic responses to competition by invasive and non-invasive congeners in the Commelinaceae. Biological Invasions, Dordrecht, v. 8, p. 797-807, 2006.

CARPANEZZI, O. T. B. Espécies vegetais exóticas no Parque Estadual de Vila Velha: subsídios para controle e erradicação. 2007. 28 f. Monografia (Especialização em Análise Ambiental) Universidade Federal do Paraná, Curitiba. 2007.

CAVAlHEIRO, A. L.; TOREZAN, J. M. D.; FADELLI. E L. Recuperação de áreas degradadas: procurando por diversidade e funcionamento dos ecossistemas. In: MEDRI, M. E.; BIANCHINI, E.; SHIBATTA, O. A.; PIMENTA, J. A. (Ed.). A bacia do rio Tibagi. Edição dos autores: Londrina. 2002. p. 213-224.

DECHOUM, M. S.; ZILLER, S. R. Métodos para controle de plantas exóticas invasoras. Biotemas, Florianópolis, v. 26, p. 6977,2013

FINE, P. V. A. The invasibility of tropical forests by exotic plants. Journal of Tropical Ecology, Winchelsea, v. 18, p. 687-705, 2002. FOXCROFT, L. C.; RICHARDSON, D. M.; WILSON, J. R. U. Ornamental plants as invasive aliens: problems and solutions in Kruger National Park, South Africa. Environmental Management, New York, v. 41, p. 32-51, 2008.

HOOPER, E.; CONDIT, R.; LEGENDRE, P. Responses of 20 native tree species to reforestation strategies for abandoned farmland in Panama. Ecological Applications, Ithaca, v. 12, n. 6, p. 1626-1641, 2002.

KELLY, D.; SKIPWORTH, J. P. Tradescantia fluminensis in a Manawatu (New Zealand) forest: I. growth and effects on regeneration. New Zealand Journal of Botany, Wellington, v. 22, p. 393-397, 1984. 
LORENZI, H.; SOUZA, H. M. Plantas ornamentais no Brasil: arbustivas, herbáceas e trepadeiras. 4 ed. São Paulo: Nova Odessa, Instituto Plantarum, 2008. $1120 \mathrm{p}$.

MACK, R. N. ; SIMBERLOFF, D.; LONSDALE, W. M.; EVANS, H.; CLOUT, M.; BAZZAZ, F. A. Biotic invasions: causes, epidemiology, global consequences, and control. Ecological Applications, Ithaca, v. 10, p. 689-710, 2000.

MARCO, A.; LAVERGNE, S.; DUTOIT, T.; BERTAUDIEREMONTES, V. From the backyard to the backcountry: how ecological and biological traits explain the escape of garden plants into Mediterranean old fields. Biological Invasions, Dordrecht, v. 12, p. 761-779, 2010.

NRCS PLANTS - NATURAL RESOURCES CONSERVATION SERVICE. Plants database. Disponível em $<$ http://www.plants. usda.gov>. Acesso em: 01 dezembro 2011.

PINTO, M. A. R.; NUNES, M. A.; DUARTE, E. B.; OLIVEIRA, C. A. Interferência da espécie exótica Tradescantia zebrina Heynh. no desenvolvimento das espécies de Anadenanthera macrocarpa (Benth.) Brenan e Piptadenia Gonoacantha (Mart.) J. F. Macbr. no Horto Florestal Anhumas, Itajubá, MG. In: CONGRESSO DE ECOLOGIA DO BRASIL, 8, 2007, Caxambu. Resumos... Caxambu: SBE, 2007. Versão eletrônica.

R DEVELOPMENT CORE TEAM. R: a language and environment for statistical computing. 2009. Disponível em $<\mathrm{http}$ ://www.Rproject.org $>$.

REJMÁNEK, M. Species richness and resistance to invasions. In: ORIANS, G.; DIRZO, R.; CUSHMAN, H. (Ed.). Biodiversity and ecosystem processes in tropical forests. Berlin: Springer-Verlag, 1996. p. 153-172.

RODOLFO, A. M.; GREGORINI, M. Z.; CANDIDO-JR, J. F.; TEMPONI, L. G. Levantamento e padrão de distribuição das plantas exóticas na trilha do Poço Preto do Parque Nacional do Iguaçu - PR. In: CONGRESSO DE ECOLOGIA DO BRASIL, 8, 2007, Caxambu. Resumos... Caxambu: CONGRESSO DE ECOLOGIA DO BRASIL, 2007. Versão eletrônica.
SIMBERLOFF, D.; HOLLE, B. V. Positive interactions of nonindigenous species: invasional meltdown? Biological Invasions, Dordrecht, v. 1, p. 21-32, 1999.

SHIBATTA, O. A. ; GALVES, W .; CARMO, W. P. D.; LIMA, I. P.; LOPES, E. V.; MACHADO, R. A. A fauna de vertebrados do campus da Universidade Estadual de Londrina, região norte do estado do Paraná, Brasil. Semina: Ciências Biológicas e da Saúde, Londrina, v. 30, n. 1, p. 3-26, 2009.

STANDISH, R. J. Experimenting with methods to control Tradescantia fluminensis, an invasive weed of native forest remnants in New Zealand. New Zealand Journal of Ecology, Wellington, v. 26, n. 2, p. 161-170, 2002.

STANDISH, R. J.; ROBERTSON, A. W.; WILLIAMS, P. A. The impact of an invasive weed Tradescantia fluminensis on native forest regeneration. Journal of Applied Ecology, London, v. 38, p. 1253-1263, 2001.

STANDISH, R. J.; WILLIAMS, P. A.; ROBERTSON, A. W.; SCOTT, N. A.; HEDDERLEY, D. I. Invasion by a perennial herb increases decomposition rate and alters nutrient availability in warm temperate lowland forest remnants. Biological Invasions, Dordrecht, v. 6, p. 71-81, 2004.

VILÀ, M.; GIMENO, I. Does invasion by an alien plant species affect the soil seed bank? Journal of Vegetation Science, Xalapa, v.18, p. 423-430, 2007.

ZANGARO, W.; NISIZAKI, S. M. A.; DOMINGOS, J. C. B.; NAKANO, E. M. Mycorrhizal response and successional status in 80 woody species from south Brazil. Journal of Tropical Ecology, Winchelsea, v. 19, p. 315-324, 2003.

ZENNI, R. D.; ZILLER, S. R. An overview of invasive plants in Brazil. Revista Brasileira de Botânica, São Paulo, v. 34, n. 3, p. 431-446, 2011. 